\title{
Chaetominine induces cell cycle arrest in human leukemia K562 and colon cancer SW1116 cells
}

\author{
JINGYUN YAO $^{1,2}$, JIANBO XIAO $^{3}$, XING WEI $^{1,2}$ and YANHUA LU ${ }^{1,2}$ \\ ${ }^{1}$ State Key Laboratory of Bioreactor Engineering; ${ }^{2}$ Shanghai Collaborative Innovation Center for \\ Biomanufacturing Technology, East China University of Science and Technology, Shanghai 200237; \\ ${ }^{3}$ Institute of Chinese Medical Sciences, State Key Laboratory of Quality Research in Chinese Medicine, \\ University of Macau, Taipa, Macau 999078, P.R. China
}

Received August 5, 2016; Accepted November 21, 2017

DOI: $10.3892 / \mathrm{ol} .2018 .9161$

\begin{abstract}
Chaetominine is a cytotoxic alkaloid that has been demonstrated to promote apoptotic cell death in human leukemia K562 cells. In the present study, chaetominine inhibited K562 ( $\left.\mathrm{IC}_{50} 34 \mathrm{nM}\right)$ and SW1116 ( $\left.\mathrm{IC}_{50} 46 \mathrm{nM}\right)$ cell growth. However, it remains unclear whether the inhibition of cell growth is associated with the cell cycle. To assess this potential relationship, the effect of chaetominine on the cell cycle of K562 and SW1116 cells was examined. Chaetominine treatment caused cell apoptosis and $\mathrm{G}_{1}$-phase arrest in SW1116 cells. Conversely, K562 cells underwent S-phase arrest according to flow cytometric analysis. The present study also aimed to elucidate the molecular mechanisms underpinning cell cycle regulation following the incubation of the associated cells with chaetominine. Western blot and reverse transcription-quantitative polymerase chain reaction analyses suggested that chaetominine treatment facilitated the expression of p53, p21, checkpoint kinase 2 (Chk2) and phosphorylated ataxia telangiectasia mutated (p-ATM) and caused a reduction in the mRNA levels of cyclin E and cyclin-dependent kinases (CDKs) 2 and 4. These results suggest that chaetominine may be involved in the regulation of p53/p21 and ATM and Rad3-related (ATM)/Chk2 signaling in SW1116 cells. Previous studies have demonstrated that these signaling pathways are responsible for $\mathrm{G}_{1}$-phase arrest. Results of the present study demonstrated that the expression of $\mathrm{p}$-ATR and Chk1 were increased in K562 cells. Additionally, cdc25A levels were decreased, while protein and gene expression levels of cyclin A and CDK2 were repressed. These results elucidated the role of chaetominine in in the regulation of ATR/cdc25A/Chk1 expression in K562 cells. These proteins
\end{abstract}

Correspondence to: Professor Yanhua Lu, State Key Laboratory of Bioreactor Engineering, East China University of Science and Technology, 130 Meilong Road, Shanghai 200237, P.R. China E-mail: luyanhua@ecust.edu.cn

Key words: cell cycle arrest, cyclin, cyclin-dependent kinase, chaetominine, K562 cells, SW1116 cells are thus important determinants in the initiation of S-phase arrest. These data support the hypothesis that chaetominine is a potential anti-cancer therapeutic agent that targets the cell cycle.

\section{Introduction}

Natural products and their respective metabolites are valuable resources that have enabled the detection of novel chemotherapeutic agents (1). Among these, alkaloids have demonstrated promising anti-cancer effects. These compounds have also been revealed to elicit effects on a variety of different targets, including regulators associated with cell cycle progression, cell apoptosis and drug resistance inhibition (2). Chaetominine is an alkaloid (Fig. 1A) isolated from the metabolites of an endophytic fungus, Aspergillus fumigatus CY018 (3). A previous study demonstrated that chaetominine may be lethal to human leukemia K562 cells, with its effects being mediated through the mitochondrial apoptosis pathway (4). Previous studies have indicated that similar compounds have the capacity to inhibit cancer cell growth by inducing cell apoptosis and/or cell cycle arrest (4-6).

Efficient regulation of the cell cycle is crucial to the process of cell survival and involves the prevention of uncontrolled cell division alongside the detection and repair of genetic damage associated with tumorigenesis (7). Checkpoints are pivotal components of the cell cycle regulative machinery and are governed by effector kinases, including ataxia telangiectasia mutated (ATM) and ATM and Rad3-related (ATR) proteins. The predominant downstream transducers of checkpoints include checkpoint kinase 1 and checkpoint kinase 2 (Chk1 and Chk2) as well as p53 (8). The activation of the p53-p21 cascade in the ATM/Chk2/p53 signaling pathway facilitates the induction of $\mathrm{G}_{1}$-phase arrest (7). Conversely, S-phase arrest is primarily triggered by multiple pathways that involve the inhibition of cell division cycle 25A (cdc25A). These pathways transmit checkpoint signals to cyclin-dependent kinases (CDKs), which form complexes with cyclins, resulting in cell cycle arrest (9). CDK2 and CDK4 are responsible for $\mathrm{G}_{1} / \mathrm{S}$ transitions during the cell cycle. These events occur following the interaction of kinases with their respective cyclin complex subunits. The binding of cyclin $E$ with $\mathrm{G}_{1}$-phase CDK2 
promotes the transition of $\mathrm{G}_{1}$-to $\mathrm{S}$-phase, while cyclin $\mathrm{A}$ is required to activate $\mathrm{CDK} 2$ for progression through the S-phase $(9,10)$. Once cell cycle arrest occurs, related signaling pathways are activated, leading to the initiation of the cell death program. This results in the inhibition of cancer cell growth. Accordingly, biomedical studies are focused on the identification and evaluation of novel inhibitors of protein kinases that are restricted to the cell cycle (2).

Chaetominine has been demonstrated to exhibit toxic effects against the human leukemia cell line K562 and the human colon cancer cell line SW1116 (11). However, the molecular mechanisms that underpin the cytotoxic effects of chaetominine are yet to be elucidated fully. Following a previous study that observed the cytotoxic and apoptotic effects of K562 cells (4), the present study hypothesized that chaetominine may alter cell cycle progression in these two cancer cell lines. The apoptotic effects induced by chaetominine on SW1116 cells and cell cycle regulation in SW1116 and K562 cells following treatment with chaetominine were also assessed. The inhibitory effects on cell growth promoted by chaetominine are likely to vary depending on the cell type that is exposed to the compound. Additionally, the molecular mechanisms involved in chaetominine-induced cell cycle arrest in K562 and SW1116 cells were elucidated in the present study.

\section{Materials and methods}

Reagents. Chaetominine was extracted from a liquid culture of A. fumigatus CY018. The purity of the preparation was determined to be $99.8 \%$ (4). MTT was purchased from Sigma-Aldrich; Merck KGaA (Darmstadt, Germany).

Cell culture. Human leukemia and colon cancer cell lines, K562 and SW1116, were obtained from the Shanghai Institute for Biological Sciences (Shanghai, China). K562 cells were cultured in RPMI-1640 (Gibco; Thermo Fisher Scientific, Inc., Waltham, MA, USA) containing $10 \%$ fetal bovine serum (FBS; Gibco; Thermo Fisher Scientific, Inc.) and SW1116 cells were cultured in Dulbecco's modified Eagle's medium (Gibco; Thermo Fisher Scientific, Inc.) containing 10\% FBS. Cells were maintained for $1-2$ days at $37^{\circ} \mathrm{C}$ in a humidified incubator containing $5 \% \mathrm{CO}_{2}$.

Cell viability assay. The effect of chaetominine on cancer cell viability was evaluated using a MTT assay. Chaetominine was dissolved in $1 \mathrm{ml}$ dimethyl sulfoxide at a concentration of $1 \mathrm{mM}$ (DMSO; Sigma-Aldrich; Merck KGaA, Darmstadt, Germany) for the following assays. Cells were seeded in 96-well plates at a concentration of $10^{5}$ cells $/ \mathrm{ml}$. All cell lines were incubated with $100,50,25,12.5,6.25$ or $0 \mathrm{nM}$ chaetominine for $48 \mathrm{~h}$, and $1 / 10,000$ (v/v, $0.01 \mu 1$ DMSO in $100 \mu 1$ reaction system) DMSO was used instead of chaetominine for the control groups. Each well was supplemented with $20 \mu \mathrm{l}$ MTT $(5 \mathrm{mg} / \mathrm{ml}$, diluted in RPMI-1640 medium to dissolve the purple formazan) and incubated for a further $4 \mathrm{~h}$ prior to testing. The absorbance was subsequently measured using a microplate reader (SpectraMax ${ }^{\circledR}$ i3; Molecular Devices LLC, Sunnyvale, CA, USA) at an experimental wavelength of $570 \mathrm{~nm}$ and a reference wavelength of $630 \mathrm{~nm}$. The half maximal inhibitory concentration $\left(\mathrm{IC}_{50}\right)$ values were calculated using GraphPad Prism 5 (GraphPad Software, Inc., La Jolla, CA, USA).

Annexin V-Fluorescein isothiocyanate (FITC)/propidium iodide $(P I)$ staining. Apoptotic rate was measured using flow cytometry. Cells were treated with 100, 50, 25 and $0 \mathrm{nM}$ chaetominine for $24 \mathrm{~h}$ in 6 -well plates. Cells were then harvested with PBS and incubated with Annexin V-FITC at $20-25^{\circ} \mathrm{C}$ for $10 \mathrm{~min}$ in the dark using an Annexin V-FITC/PI kit (Nanjing Keygen Biotech Co., Ltd., Nanjing, China). Cells were subsequently suspended in a mixture of Annexin $\mathrm{V}$ and PI buffer following centrifugation at room temperature, at a speed of $200 \mathrm{x} \mathrm{g}$ for $5 \mathrm{~min}$. Apoptotic cells were analyzed using a flow cytometer (FACSAria; BD Biosciences, San Jose, CA, USA). Annexin-V FITC positive and PI negative results indicated early apoptosis, while Annexin-V FITC positive and PI positive results indicated late apoptosis. The FACSAria was equipped with BD FACSDiva software v6.0 (BD Biosciences).

Cell cycle assay. The effects of chaetominine on cell cycle progression were analyzed using a Cell Cycle Detection kit (Nanjing Keygen Biotech Co., Ltd.). Cells were treated with $40 \mathrm{nM}$ chaetominine for $0,12,24$ of $48 \mathrm{~h}$, or $40,20,10$ or $0 \mathrm{nM}$ chaetominine for $24 \mathrm{~h}$. Following treatment, cells were washed with PBS and fixed overnight at $4^{\circ} \mathrm{C}$ in $70 \%$ ethanol. Cells were then incubated with $100 \mu \mathrm{l}$ RNase at $37^{\circ} \mathrm{C}$ for $30 \mathrm{~min}$ and stained for $30 \mathrm{~min}$ in the dark with $400 \mu \mathrm{l}$ PI solution at $4^{\circ} \mathrm{C}$. The percentage of cells in different phases was monitored using a flow cytometer (FACSAria; BD Biosciences) at $488 \mathrm{~nm}$. The FACSAria was equipped with BD FACSDiva software v6.0 (BD Biosciences).

Western blot assay. Whole lysates were prepared with cell lysis buffer (Biotech Well, Shanghai, China) following incubation with 40, 20, 10 or $0 \mathrm{nM}$ chaetominine for $24 \mathrm{~h}$. Lysates were subsequently washed with PBS and protein concentrations were determined using a bicinchoninic acid protein assay kit (Pierce; Thermo Fisher Scientific, Inc.). Protein samples $(20 \mu \mathrm{g} /$ lane $)$ containing $0.01 \%$ bromophenol blue were separated using $10 \%$ SDS-PAGE and were subsequently transferred onto polyvinylidene difluoride membranes (EMD Millipore, Billerica, MA, USA). The PVDF membranes were blocked at room temperature for $2 \mathrm{~h}$ with $5 \%$ bovine serum albumin (diluted in TBS-Tween-20; TBS-T) and incubated overnight with the appropriate primary antibody (diluted with blocking buffer) at $4^{\circ} \mathrm{C}$. Primary antibodies against ATM (dilution, 1:500; cat. no. SC-377239), phosphorylated (p)-ATM (Ser1981; dilution, 1:500; cat. no. SC-47739), Chk1 (dilution, 1:200; cat. no. SC-8408), Chk2 (dilution, 1:200; cat. no. SC-5278), p53 (dilution, 1:200; cat. no. SC-126), p21 (dilution, 1:200; cat. no. SC-469), GAPDH (dilution, 1:1,000; cat. no. SC-69778) and $\beta$-actin (dilution, 1:1,000; cat. no. SC-47778) were obtained from Santa Cruz Biotechnology, Inc. (Dallas, TX, USA). ATR (dilution, 1:1,000; cat. no. 2790), p-ATR (Ser428; dilution, 1:1,000; cat. no. 2853), Cdc25A (dilution, 1:1,000; cat. no. 3652), cyclin A (dilution, 1:1,000, cat. no. 4656) and CDK2 (dilution, 1:1,000; cat. no. 2546) primary antibodies were obtained from Cell Signaling Technology, Inc. (Danvers, MA, USA). Horseradish peroxidase-conjugated goat anti-mouse (dilution, 1:1,000; cat. no. A0216) and goat 
anti rabbit (dilution, 1:1,000; cat. no. A0208) secondary IgG $(\mathrm{H}+\mathrm{L})$ antibodies were purchased from Beyotime Institute of Biotechnology (Haimen, China). Following three sequential TBS-T washes, the membranes were incubated overnight with horseradish peroxidase-labeled secondary antibodies at $4^{\circ} \mathrm{C}$. An enhanced chemiluminescence kit (Biotech Well) was used to visualize the immunoreactions. Protein levels were determined following the detection of chemiluminescent signals. The signals were quantified using Quantity One v4.62 software (Bio-Rad Laboratories Inc., Hercules, CA, USA).

Reverse transcription-quantitative polymerase chain reaction $(R T-q P C R)$. Total RNA was extracted using a Total RNA Extraction kit (Nanjing Keygen Biotech Co., Ltd.). RNA samples were quantified using UV spectrophotometry at a wavelength of $260 \mathrm{~nm}$. Reverse transcription into cDNA was performed using a PrimeScript ${ }^{\mathrm{TM}}$ RT reagent kit (Takara Biotechnology Co., Ltd, Dalian, China) with 500 ng total RNA. cDNA was subsequently utilized for PCR analysis. The qPCR reaction was conducted using SYBR ${ }^{\circledR}$ Premix Ex Taq ${ }^{\mathrm{TM}}$ II (Takara Biotechnology Co., Ltd.) on a CFX96 Touch ${ }^{\mathrm{TM}}$ PCR Detection system (Bio-Rad Laboratories, Inc.). The conditions for PCR amplification were as follows: 40 cycles of initial duration at $94^{\circ} \mathrm{C}$ for $30 \mathrm{sec}$, annealing at $60^{\circ} \mathrm{C}$ for $30 \mathrm{sec}$ and extension at $72^{\circ} \mathrm{C}$ for $1 \mathrm{~min}$. The primer sequences used were as follows: Cyclin A, 5'-TCC ATGTCAGTGCTGAGAGGA-3' (forward), 5'-GAAGGTCCA TGAGACAAGGC-3' (reverse); CDK2, 5'-GCTTTCTGCCAT TCTCATCG-3' (forward), 5'-GTCCCCAGAGTCCGAAAG AT-3' (reverse); cyclin E, 5'-TTTCTTGAGCAACACCCT-3' (forward), 5'-GTCACATACGCAAACTGG-3' (reverse); CDK4, 5'-CTGAGAATGGCTACCTCTCGATATG-3' (forward), 5'-AGAGTGTAACAACCACGGGTGTAAG-3' (reverse); glyceraldehyde-3-phophate dehydrogenase (GAPDH), 5'-CAA CGGATTTGGTCGTATT-3' (forward), 5'-CACAGTCTTCTG GGTGGC-3' (reverse). The mRNA level associated with each gene was normalized to that of the internal control, GAPDH, and was quantified using the $-2^{\Delta \Delta \mathrm{Cq}}$ method (12).

Statistical analysis. Data were analyzed using Graphpad Prism 5 (Graphpad Software Inc., La Jolla, CA, USA) and are expressed as the mean \pm standard deviation. Group comparisons of experimental data were performed using a one-way analysis of variance with post-hoc Newman-Keuls tests. $\mathrm{P}<0.05$ was considered to indicate a statistically significant result.

\section{Results}

Chaetominine inhibits the cell viability of K562 and SW1116 cells. The cytotoxic effects of chaetominine on K562 and SW1116 cells were analyzed using an MTT assay. The $\mathrm{IC}_{50}$ values for chaetominine in K562 and SW116 cells were $33.7 \pm 0.2$ and $45.9 \pm 3.4 \mathrm{nM}$, respectively. The results demonstrated that chaetominine inhibited K562 and SW1116 cell viability at concentrations of $6.25,12.5,25,50$ and $100 \mathrm{nM}$ (Fig. 1B). These data were consistent with those reported in a previous study (11).

Chaetominine induces apoptosis of SW1116 cells. A previous study demonstrated that chaetominine induces apoptotic cell

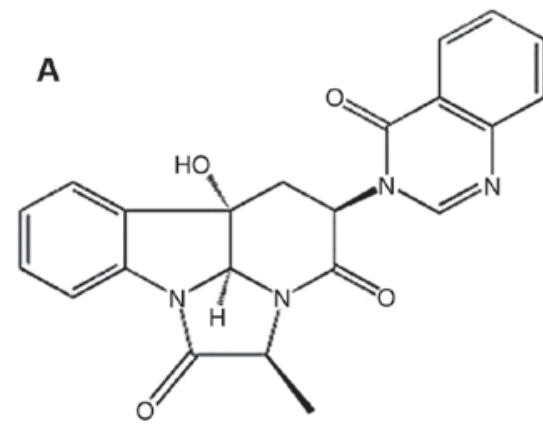

B

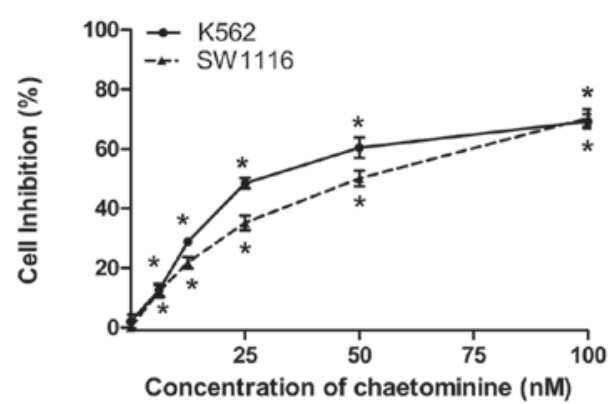

Figure 1. (A) Chemical structure of chaetominine. (B) Chaetominine inhibited K562 and SW1116 cell proliferation. Cells were incubated with 100, 50, $25,12.5,6.25$ or $0 \mathrm{nM}$ chaetominine for $48 \mathrm{~h}$ and were tested using MTT. The rate of cell inhibition is expressed as the mean \pm standard deviation following three independent experiments. ${ }^{*} \mathrm{P}<0.05$ vs. $0 \mathrm{nM}$ for each respective group.

death in K562 cells (4). Following these results, the present study utilized flow cytometry to assess whether chaetominine induces cell apoptosis in SW1116 cells. The apoptosis rates were determined using dual staining with Annexin V-FITC and PI. It was revealed that chaetominine significantly increased early and late apoptosis rates compared with the control group (Fig. 2A). The total apoptosis rate gradually increased from $6.4 \%$ in the control group to $16.4,35.6$ and $60.0 \%$ in cells incubated with 25,50 and $100 \mathrm{nM}$ chaetominine, respectively. These results suggested that chaetominine induces apoptotic cell death in SW1116 cells.

Chaetominine results in $G_{1}$ or $S$ phase cell cycle arrest. To elucidate whether the cell cycle was involved in the inhibition of cell growth following chaetominine treatment, K562 and SW1116 cells were treated with chaetominine to facilitate the detection of cell cycle phase distribution. The results demonstrated that chaetominine treatment resulted in an accumulation of $\mathrm{G}_{1}$-phase SW1116 cells (control, 51.84\%; $12 \mathrm{~h}$, $54.45 \%$; 24 h, 56.55\%; and 48 h, 68.78\%) in a time-dependent manner (Fig. 2B). In addition, chaetominine caused an increase in the number of S-phase K562 cells (Fig. 3A; control, 25.26\%; $10 \mathrm{nM}, 36.14 \%$; $20 \mathrm{nM}, 43.54 \%$; and $40 \mathrm{nM}, 49.5 \%$ ). This occurred in a dose-dependent manner. These results indicated that cell cycle arrest was a mechanism used by chaetominine to induce cytotoxicity in K562 and SW1116 cells. In addition, chaetominine treatment resulted in cell cycle arrest at the $\mathrm{G}_{1}$ stage (SW1116) or S stage (K562) depending on which cell type was analyzed.

Chaetominine influences the expression of ATM/Chk2/p53/p21 in SW1116 cells. In order to elucidate the 

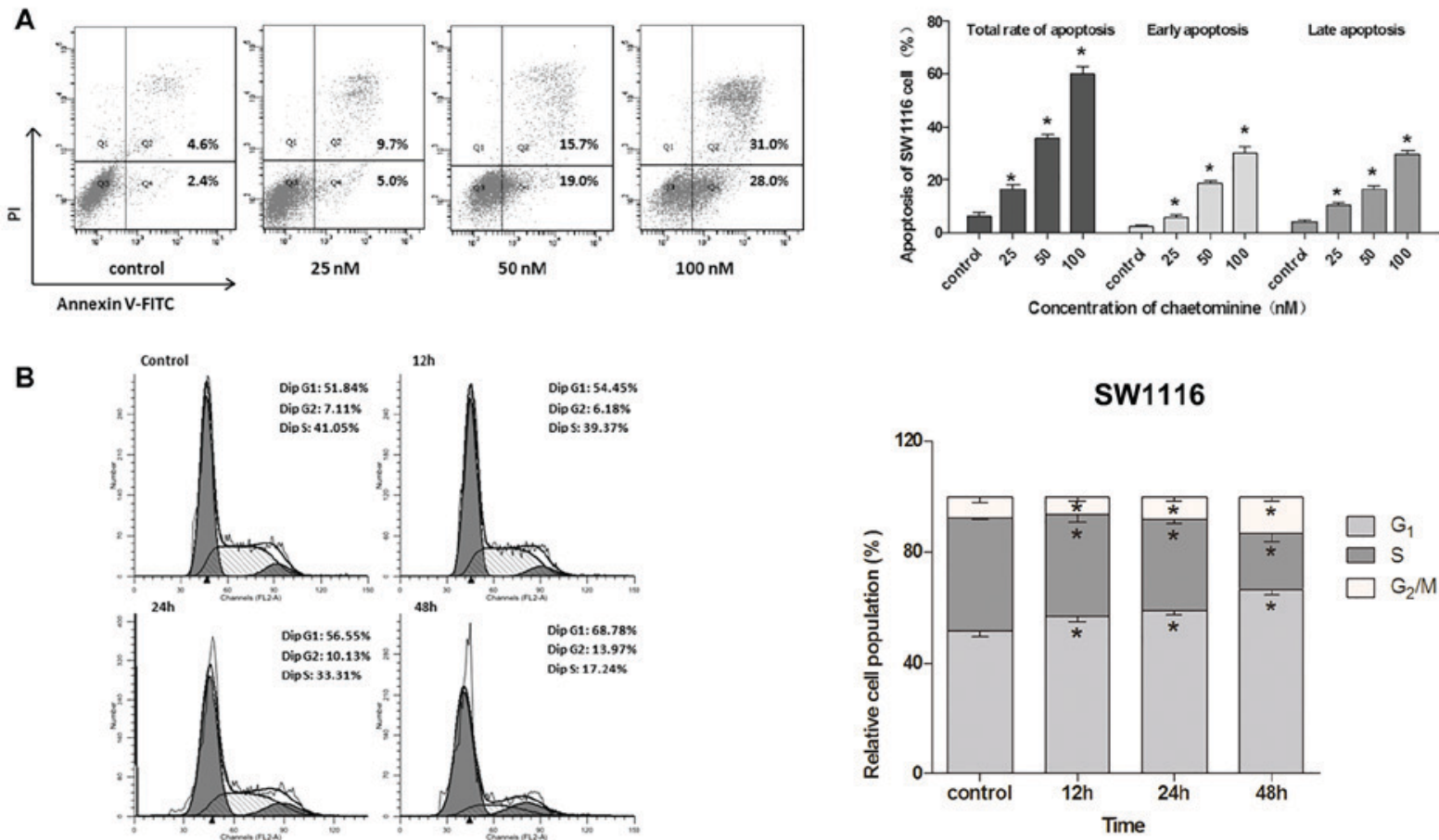

SW1116

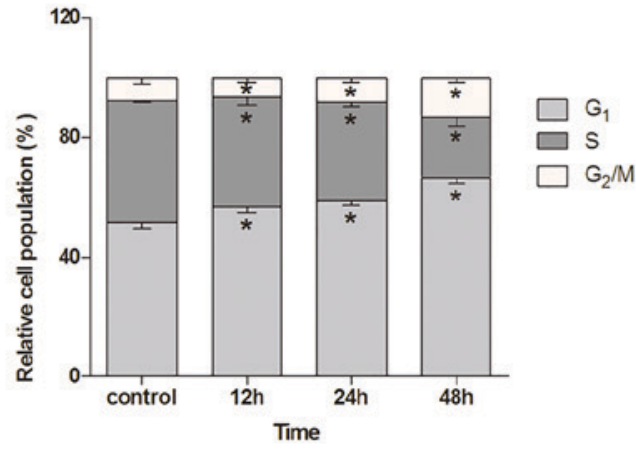

Figure 2. Chaetominine induced apoptosis and $\mathrm{G}_{1}$-phase arrest in SW1116 cells. (A) Apoptosis rates were assessed using flow cytometry. Cells were incubated in the presence or absence of 25,50 and $100 \mathrm{nM}$ chaetominine for $24 \mathrm{~h}$ prior to analysis. The total apoptotic percentages represent the average values generated following aggregation of both early and late apoptosis rates. (B) Cell cycle distribution as determined by flow cytometry. Cells were treated with $40 \mathrm{nM}$ chaetominine for $0,12,24$ or $48 \mathrm{~h}$. The percentage of cells at each phase was determined following the execution of three independent experiments. ${ }^{*} \mathrm{P}<0.05$ vs. control group. Ctrl, control; FITC, Fluorescein isothiocyanate; PI, propidium iodide.

A
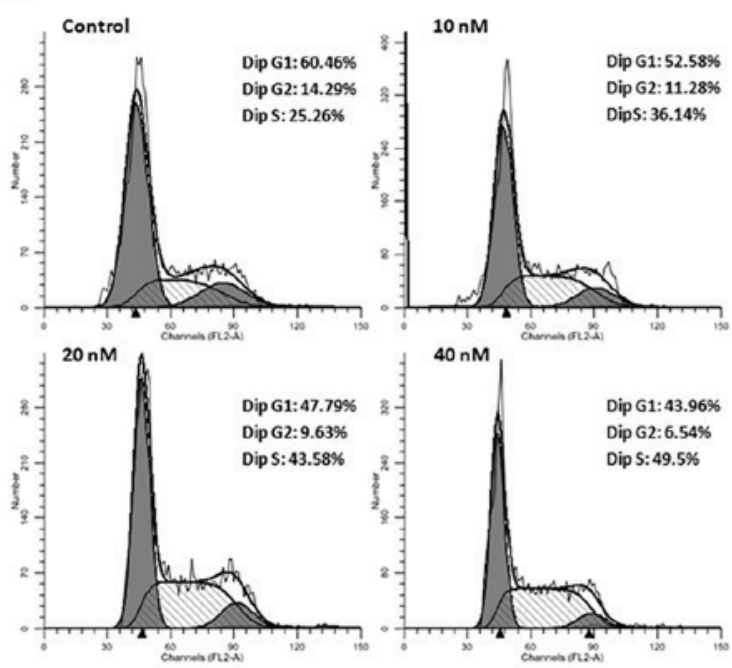

B

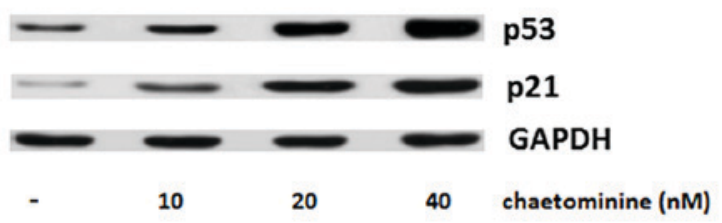

Dip G1: 52.58\% Dip 62: 11.28\% DipS: $36.14 \%$
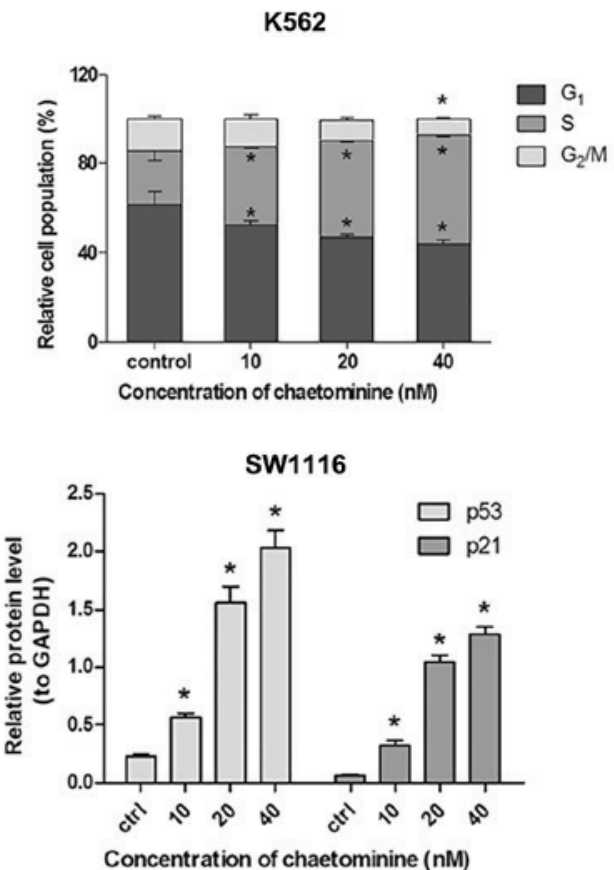

Figure 3. (A) Chaetominine induced S-phase arrest in K562 cells. Cells were untreated or treated with 10, 20 and $40 \mathrm{nM}$ chaetominine for 24 h. Cell cycle distribution was detected using flow cytometry. The percentage of cells at each phase was determined following the execution of three independent experiments. (B) Chaetominine treatment caused p53 and p21 expression increase in SW1116. Results of western blotting following incubation with antibodies against p53 and p21. The proteins used during this analysis were extracted from cells that had been untreated or treated with 10,20 or 40 nM of chaetominine for $24 \mathrm{~h}$. The bar graphs represent densitometric analysis of the protein expression levels relative to the internal reference protein, GAPDH. Data are presented as the mean \pm standard deviation $(\mathrm{n}=3)$. ${ }^{*} \mathrm{P}<0.05$ vs. control group. 
molecular mechanism associated with $\mathrm{G}_{1}$-phase arrest exhibited by chaetominine treated SW1116 cells, the signaling proteins involved in the regulation cascade of the cell cycle were evaluated using western blotting. p53 induces cell cycle arrest at the $\mathrm{G}_{1}$-stage, resulting in the activation of $\mathrm{p} 21$, which promotes the inhibition of CDKs (2). The results demonstrated that the expression of p53 and p21 were upregulated in SW1116 cells following incubation with chaetominine (Fig. 3B). This upregulation occurred in a time-dependent manner. The association between ATM/Chk2 and chaetominine was assessed. It was demonstrated that chaetominine increased the phosphorylation and accumulation of ATM and Chk2, respectively. However, treatment did not notably affect the expression of ATM (Fig. 4A). The significant phosphorylation of ATM is accompanied by the upregulation of Chk2 expression and demonstrates that pATM is associated with Chk1 regulation, which assists in checkpoint regulation (13). It was determined that chaetominine modulates the ATM/Chk2/p53/p21 signaling pathway, which is an important effector of $\mathrm{G}_{1}$-phase arrest $(7,13)$.

Chaetominine alters the expression of ATR/Chk1/cdc25A in K562 cells. The mechanism by which chaetominine induces S-phase arrest in K562 cells was further assessed. Western blotting was utilized to determine if the effects elicited by chaetominine were associated with the alteration of cell-cycle regulatory kinases. Cyclin A is a critical component involved in the regulation of cell cycle progression, which becomes functionally active once bound to CDK2. This association subsequently allows cells to continue through the S-phase (14). Based on previous data, the present study determined the expression levels of cyclin A, CDK2 and upstream proteins including cdc25A, Chk1/2 and ATR/p-ATR in K562 cells. The results of the present study demonstrated that cyclin A and CDK2 expression were significantly reduced by chaetominine compared with the control (Fig. 4B). In addition, K562 cells expressed significantly higher levels of Chk1 $(\mathrm{P}<0.05$ at $10,20$ and $40 \mu \mathrm{M})$ and $\mathrm{Chk} 2(\mathrm{P}<0.05$ at $40 \mu \mathrm{M})$ following treatment with chaetominine compared with the control (Fig. 4C). Furthermore, a detectable alteration in Chk2 levels was only observed following $40 \mathrm{nM}$ chaetominine treatment, while the expression of Chk1 changed significantly even at the lowest concentration $(10 \mathrm{nM})$ of chaetominine (Fig. 4C). Chk1 protein levels were 5.7-fold greater following $40 \mathrm{nM}$ chaetominine treatment compared with controls, while the Chk2 protein levels had increased by 1.5 -fold when the same treatment was applied. The level of cdc $25 \mathrm{~A}$ was marginally attenuated following $40 \mathrm{nM}$ chaetominine treatment (to $\sim 50 \%$ that of the control group; Fig. 4C). The results of the present study suggest that an elevation in p-ATR expression occurred following treatment with chaetominine. These results suggest that chaetominine treatment upregulated p-ATR and Chk1 protein levels and downregulated cdc25A, cyclin A and CDK2, and these proteins are reported to participate in the initiation of S-phase arrest $(14,15)$.

Chaetominine treatment affects cell cycle regulator cyclin $A / E$ and CDK2/4 mRNA levels. To assess the role of direct regulators of cell cycle arrest in K562 and SW1116 cells, the mRNA levels of specific genes were determined using RT-qPCR. The results demonstrated that the mRNA levels of cyclin A and CDK2 in K562 cells were significantly decreased following incubation with $40 \mathrm{nM}$ chaetominine (Fig. 5). However, minimal changes in the mRNA levels of cyclin E or CDK4 were observed, when compared with the control groups. These results are consistent with the cyclin A and CDK2 expression variation trends observed in chaetominine-treated K562 cells in the present study. Conversely, chaetominine resulted in the downregulation of cyclin E, CDK2 and CDK4 in SW1116 cells. However, the same effect was not observed in cyclin A. These results suggested that chaetominine treatment altered the mRNA levels of cyclin E, CDK2 and CDK4 in SW1116 cells as described in Fig. 6.

\section{Discussion}

Among natural anti-cancer alkaloids, those derived from marine metabolites and their derivatives may be a vital resource for chemotherapeutic discovery due to a low effective dosage and increased selectivity $(2,16)$. Previous studies pertaining to these benefits have served a crucial role in the investigation of anticancer candidates in natural products. To further aid in these investigations, the present study utilized the fungal metabolite chaetominine isolated from a culture of Aspergillus fumigatus CY018 (3). This compound exhibited cytotoxic effects in two human cancer cell lines (K562 and SW1116 cells) when used in nanomolar concentrations. However, the effects of chaetominine on the regulation of the cell cycle, which is an important component involved in controlling cellular proliferation, are not well understood. The current study was performed to investigate the relative contribution of chaetominine to the molecular mechanisms associated with cell cycle regulation in K562 and SW1116 cells.

The present study determined that chaetominine markedly inhibited cell growth in $\mathrm{K} 562\left(\mathrm{IC}_{50} ; 34 \mathrm{nM}\right)$ and SW1116 $\left(\mathrm{IC}_{50} ; 46 \mathrm{nM}\right)$ cells. These results are consistent with those of previous studies, which indicated that chaetominine may be a candidate for anti-cancer treatment $(11,15)$. The results of the present study, including the dose-dependent increase in apoptosis rate and a sub- $\mathrm{G}_{1}$ peak in the SW1116 cells treated with $40 \mathrm{nM}$ chaetominine for $24 \mathrm{~h}$, demonstrate that the cytotoxic role of chaetominine in SW1116 and K562 cells is associated with the induction of apoptosis. However, no obvious sub- $\mathrm{G}_{1}$ peak following exposure to higher concentrations of chaetominine resulted in an inability to collect all the fragments released by dead cells. The cell cycle is an important regulatory mechanism associated with cell growth and proliferation $(7,8)$. A variety of anti-cancer agents target the abnormal growth of cells by disrupting cell cycle progression and/or inducing apoptosis (5). In the present study, SW1116 cells underwent $\mathrm{G}_{1}$-phase cell cycle arrest, while K562 cells underwent $\mathrm{S}$-phase arrest following chaetominine treatment. These results suggest that the role of chaetominine in cancer cell death is dependent on cell cycle arrest and apoptosis.

Cyclins and CDKs have been demonstrated to function in the direct regulation of the cell cycle (9). RT-qPCR analysis utilized in the present study demonstrated that chaetominine-induced cell cycle arrest in K562 cells was associated with the downregulation of cyclin A and CDK2. In SW1116 
A

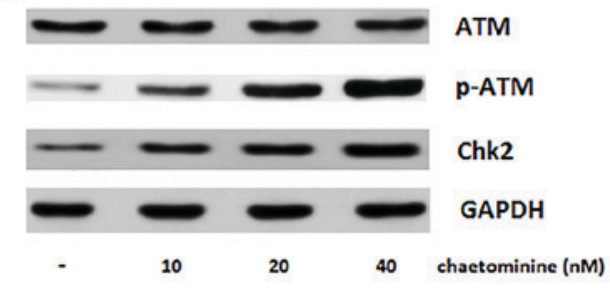

B

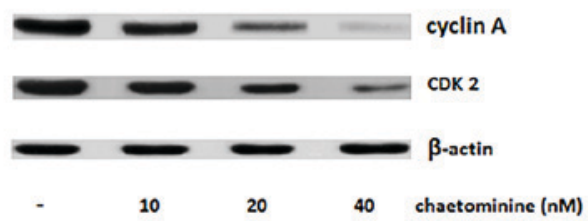

C

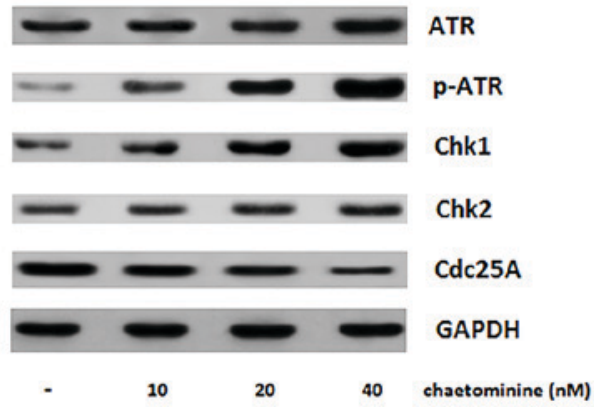

SW1116
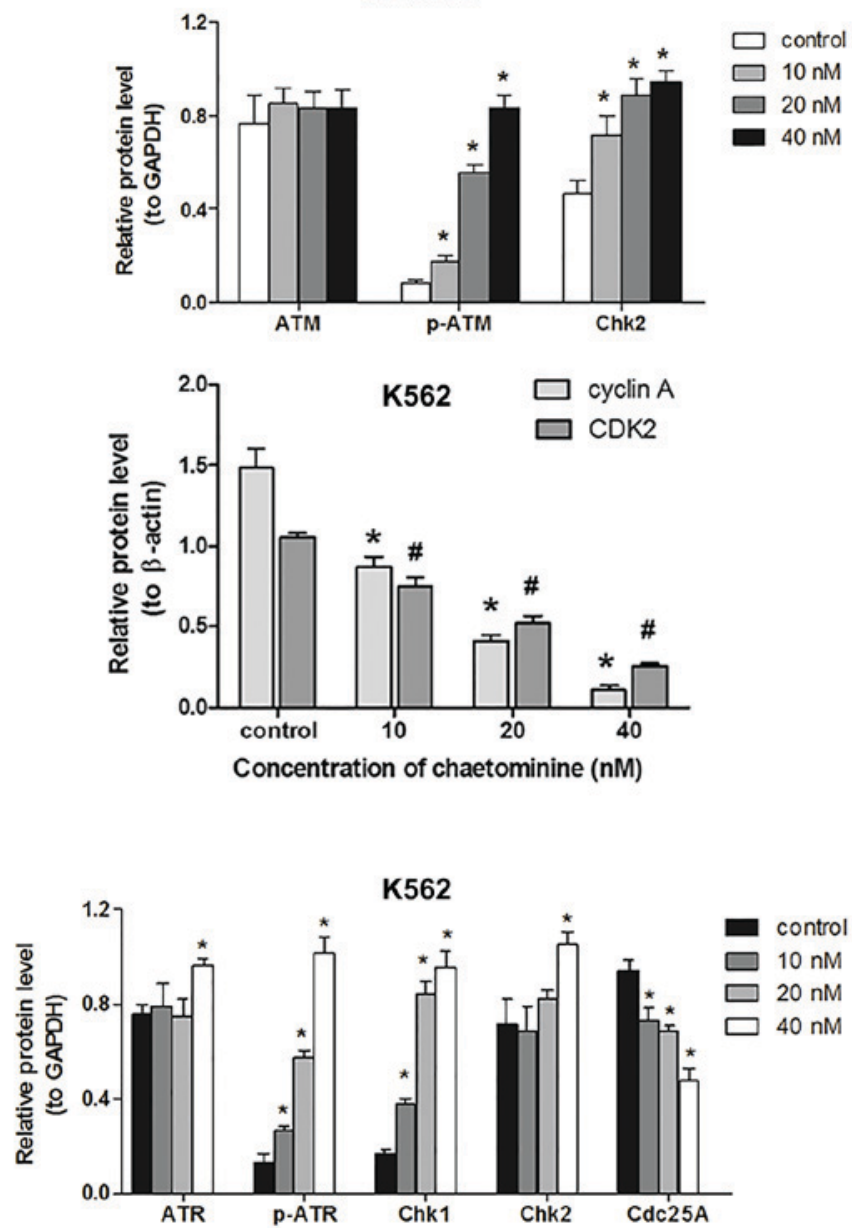

Figure 4. Modulation of signal molecules involved in chaetominine-induced cell cycle arrest. SW1116 and K562 cells were exposed to 0 , 10,20 and 40 nM chaetominine for $24 \mathrm{~h}$. (A) Protein levels following western blotting using antibodies against ATM, p-ATM (Ser1981) and Chk2. The analysis was conducted on protein extracts from SW1116 cells. * $\mathrm{P}<0.05$ vs. respective control group. (B) Results of western blotting following incubation with antibodies against cyclin A and CDK 2 of K562 cells. The protein expression levels were analyzed following densitometric analysis and results were compared to the $\beta$-actin loading control. Representative results for each experiment are presented in A and B. Data are presented as the mean \pm standard deviation ( $\mathrm{n}=3$ ) ${ }^{*} \mathrm{P}<0.05$ vs. cyclin $\mathrm{A}$ controls; ${ }^{\text {P }}<0.05$ vs. CDK2 controls. (C) Protein levels following western blotting using antibodies against ATR, p-ATR (Ser428), Chk1, Chk2 and cdc25A in K562 cells. The bar graphs represent a densitometric analysis conducted to determine protein expression relative to the internal reference protein, GAPDH. Representative blots from three independent experiments are presented and data are presented as the mean \pm standard deviation ( $\mathrm{n}=3$ ). $\mathrm{P}<0.05$ vs. respective control group. ATM, ataxia telangiectasia mutated; ATR, ATM and Rad3-related; p-ATR, phosphorylated ATR; CDK2, cyclin-dependent kinase 2; Chk, checkpoint kinase; cdc25A, cell division cycle 25A.

cells, this occurrence was accompanied by a decrease in cyclin E, CDK2, and to a lesser extent, CDK4. CDK2 activity is restricted to the $G_{1}-S$ phase of the cell cycle and results in the binding of different cyclin partners. Cyclin E/CDK2 and CDK4 are recruited for the transition from the G1-phase to the $\mathrm{S}$ phase, while cyclin $\mathrm{A} / \mathrm{CDK} 2$ is utilized for progression through the S-phase. Cyclin A and cyclin E overexpression is commonly observed in leukemia and colon cancer cells, respectively (14). This overexpression may be indicative of the different types of cell cycle arrest observed in the two cancer cell lines utilized in the present study following treatment with chaetominine. Further studies are required to elucidate whether chaetominine acts as a pharmacological inhibitor of CDKs, with a potent anti-cancer activity.

Cell cycle checkpoint pathways are crucial regulatory machineries involved in the determination of cellular responses to cancer therapy (8). ATM/Chk2 and ATR/Chk1 signaling modules are involved in the control of checkpoint networks and promote delays in the cell cycle at the $G_{1}, S$ or $G_{2}$ phase $(7,13)$. p53 is a key substrate of the ATM/Chk2 module and also acts as a pro-apoptotic protein (17). The accumulation of $\mathrm{p} 53$ results in the activation of $\mathrm{p} 21$, which inactivates the cyclin E/CDK 2 complex, subsequently leading to $\mathrm{G}_{1}$-phase arrest. This is consistent with results of the present study. It was demonstrated that SW1116 cells expressed higher levels of p-ATM, Chk2, p53 and p21 following chaetominine treatment and that these molecules were important mediators in the arrest of cells at the $G_{1}$-phase $(13,18)$. The ATR/Chk2 module elicits its effects following an increase in Chk2 activity, which causes the attenuation of cdc25, cyclin A and CDK2, thus leading to S-phase blockade (19). In K562 cells, these effects resulted in an increase in the expression of p-ATR and Chk2 following incubation with chaetominine. Conversely, the protein level of cdc25A, cyclin A and CDK2 


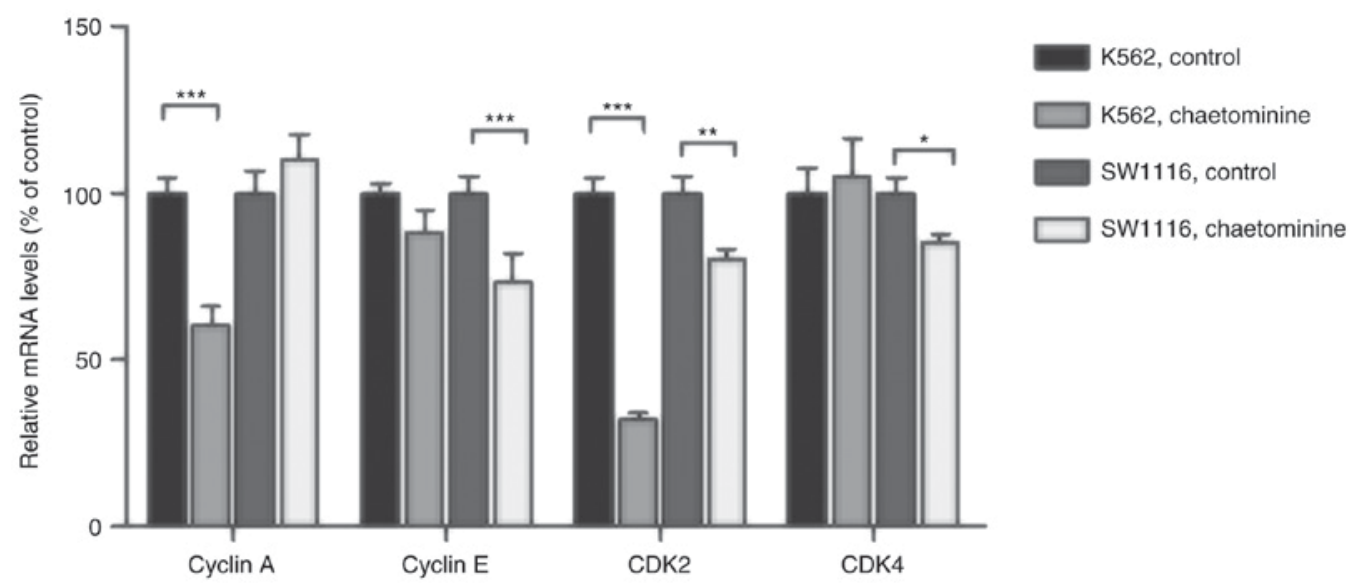

Figure 5. Role of chaetominine in the regulation of mRNA levels of cyclin A, cyclin E, CDK2 and CDK4 in K562 and SW1116 cells. K562 and SW1116 cells were incubated in the presence or absence of $40 \mathrm{nM}$ chaetominine for $24 \mathrm{~h}$ prior to reverse transcription polymerase chain reaction. Relative fold changes in comparison with the control group were calculated using the $\Delta \Delta \mathrm{C}(\mathrm{q})$ method $(\mathrm{n}=3)$. Data are presented as the mean \pm standard deviation. ${ }^{*} \mathrm{P}<0.05$, ${ }^{* *} \mathrm{P}<0.01$ and ${ }^{* * * *} \mathrm{P}<0.001$ vs. the control groups. CDK, cyclin-dependent kinase.

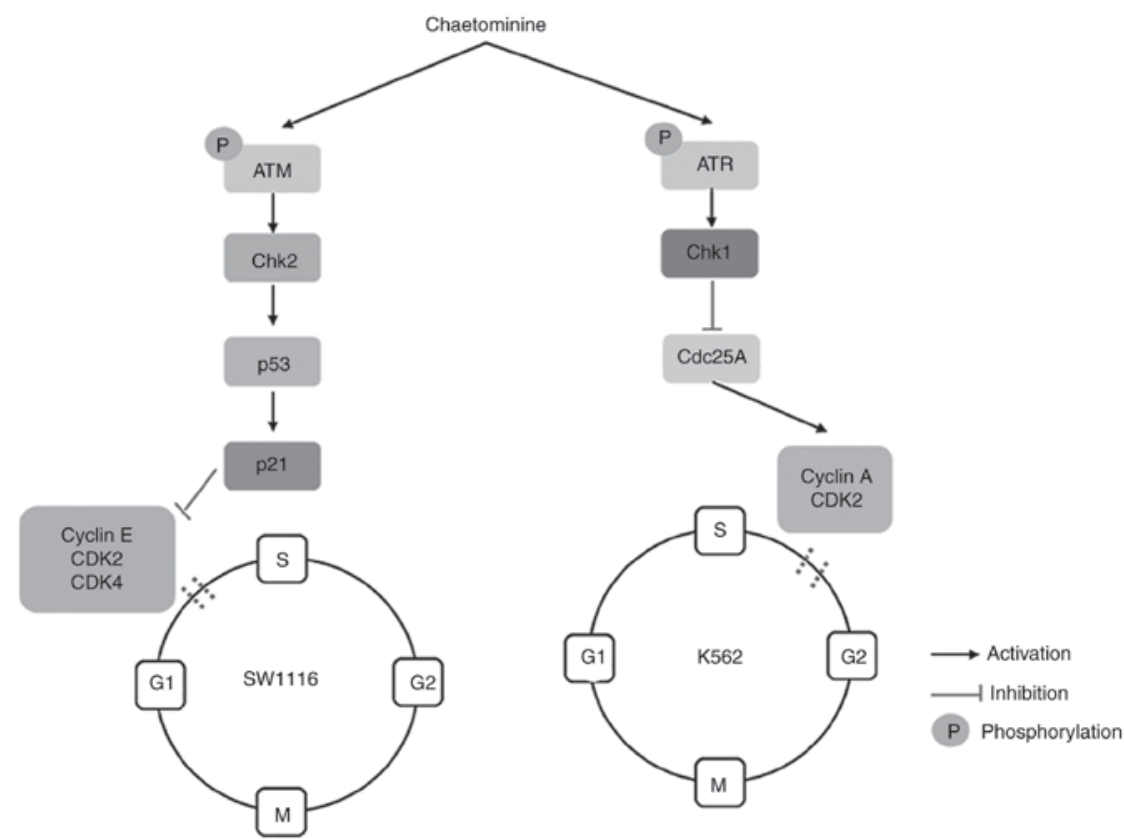

Figure 6. Pathway diagram presenting chaetominine mode of action associated with cell cycle regulation in SW1116 and K562 cells. P, phosphorylated; ATM, ataxia telangiectasia mutated; ATR, ATM and Rad3-related; Chk, checkpoint kinase; cdc25A, cell division cycle 25A; CDK, cyclin-dependent kinase.

decreased in chaetominine-treated K562 cells. However, Chk 1 protein levels remained relatively stable following incubation with the same compound. These results are consistent with those of the present study, suggesting that chaetominine affected the ATR-Chk2-cdc25A-cyclin A/CDK2 signaling pathway. This also occurred independently of $\mathrm{p} 53$, facilitating the occurrence of cell cycle arrest during the S-phase $(17,19)$.

In conclusion, the present study demonstrated that chaetominine causes the inhibition of cell growth through apoptosis and cell cycle arrest in K562 and SW1116 cells. The current study also revealed the mechanism of action that underpins cell cycle arrest following chaetominine treatment. However, it remains unclear whether the cellular response to chaetominine is actually associated with DNA damage caused by ATM or ATR signal initiation (20). Furthermore, all of the experiments conducted in the present study were performed in vitro. Further in vivo studies are therefore required to fully characterize the effects associated with this compound. Nevertheless, chaetominine may serve as an important chemotherapeutic agent with several putative clinical applications.

\section{Acknowledgements}

The present study was supported by the National High Technology Research and Development Program of China (grant no. 2013AA092901) and partially financed by the Fundamental Research Funds for the Central Universities (grant no. WF1113010) and the National Special Fund for 
State Key Laboratory of Bioreactor Engineering (grant no. 2060204).

\section{References}

1. Hussain S, Fareed S, Ansari S and Khan S: Marine natural products: A lead for anti-cancer. Indian J Geo Mar Sci 41: 27-39, 2012.

2. Imperatore C, Aiello A, D'Aniello F, Senese M and Menna M: Alkaloids from marine invertebrates as important leads for anticancer drugs discovery and development. Molecules 19 20391-20423, 2014.

3. Lu YH, Zhu YX, Jiao RH, Tan RX, Yao LY and Hu WW: Method and medium for producing Fumigaclavine $\mathrm{C}$ by Aspergillus fumigatus Fermentation. China patent, CN 103849663 B. Filed January 10, 2014; issued May 4, 2016.

4. Yao J, Jiao R, Liu C, Zhang Y, Yu W, Lu Y and Tan R: Assessment of the cytotoxic and apoptotic effects of chaetominine in a human leukemia cell line. Biomol Ther (Seoul) 24 147-155, 2016

5. Mahata S, Bharti AC, Shukla S, Tyagi A, Husain SA and Das BC: Berberine modulates AP-1 activity to suppress HPV transcription and downstream signaling to induce growth arrest and apoptosis in cervical cancer cells. Mol Cancer 10: 39, 2011.

6. Li S, Lei Y, Jia Y, Li N, Wink M and Ma Y: Piperine, a piperidine alkaloid from Piper nigrum re-sensitizes P-gp, MRP1 and BCRP dependent multidrug resistant cancer cells. Phytomedicine 19: 83-87, 2011.

7. Kastan MB and Bartek J: Cell-cycle checkpoints and cancer. Nature 432: 316-323, 2004.

8. Kerzendorfer C and O'Driscoll M: Human DNA damage response and repair deficiency syndromes: Linking genomic instability and cell cycle checkpoint proficiency. DNA Repair (Amst) 8: 1139-1152, 2009.
9. Sánchez-Martínez C, Gelbert LM, Lallena MJ and de Dios A: Cyclin dependent kinase (CDK) inhibitors as anticancer drugs. Bioorg Med Chem Lett 25: 3420-3435, 2015.

10. Poi MJ, Knobloch TJ, Sears MT, Uhrig LK, Warner BM, Weghorst CM and Li J: Coordinated expression of cyclin-dependent kinase-4 and its regulators in human oral tumors. Anticancer Res 34: 3285-3292, 2014.

11. Jiao RH, Xu S, Liu JY, Ge HM, Ding H, Xu C, Zhu HL and Tan RX: Chaetominine, a cytotoxic alkaloid produced by endophytic Chaetomium sp. IFB-E015. Org Lett 8: 5709-5712, 2006.

12. Livak KJ and Schmittgen TD: Analysis of relative gene expression data using real-time quantitative PCR and the 2(-Delta Delta C(T)) method. Methods 25: 402-408, 2001.

13. Smith J, Tho LM, Xu N and Gillespie DA: The ATM-Chk2 and ATR-Chk1 pathways in DNA damage signaling and cancer. Adv Cancer Res 108: 73-112, 2010.

14. Yam CH, Fung TK and Poon RY: Cyclin A in cell cycle control and cancer. Cell Mol Life Sci 59: 1317-1326, 2002.

15. Berthet C, Raj K, Saudan P and Beard P: How adeno-associated virus Rep78 protein arrests cells completely in $\mathrm{S}$ phase. Proc Natl Acad Sci USA 102: 13634-13639, 2005.

16. Kshirsagar UA: Recent developments in the chemistry of quinazolinone alkaloids. Org Biomol Chem 13: 9336-9352, 2015.

17. Wahl GM and Carr AM: The evolution of diverse biological responses to DNA damage: Insights from yeast and p53. Nat Cell Biol 3: E277-E286, 2001.

18. Zhang J, Ghio AJ, Gao M, Wei K, Rosen GD and Upadhyay D: Ambient particulate matter induces alveolar epithelial cell cycle arrest: Role of G1 cyclins. FEBS Lett 581: 5315-5320, 2007.

19. Bartek J, Lukas C and Lukas J: Checking on DNA damage in $S$ phase. Nat Rev Mol Cell Biol 5: 792-804, 2004.

20. Zuco V, Benedetti V and Zunino F: ATM- and ATR-mediated response to DNA damage induced by a novel camptothecin, ST1968. Cancer Lett 292: 186-196, 2010. 